\title{
Erratum to Development of a core outcome set for orthodontic trials using a mixed-methods approach: protocol for a multicentre study
}

Aliki Tsichlaki ${ }^{1 *}$, Kevin O'Brien², Ama Johal ${ }^{1}$, Zoe Z. Marshman³, Philip P. Benson ${ }^{3}$, Fiorella B. Colonio Salazar ${ }^{1,4}$ and Padhraig S. Fleming ${ }^{1}$

\section{Erratum}

In the original publication [1] have two author names errors. The original article has been updated to rectify this error. The correct versions of the author names can be found below.

Incorrect:

Zoe Z. Marshman

Philip P. Benson

Correct:

Zoe Marshman

Philip Benson

\begin{abstract}
Author details
'Department of Orthodontics, Barts and the London School of Medicine and Dentistry, Queen Mary University of London, London E1 1BB, UK. ${ }^{2}$ Division of Dentistry, Faculty of Biology, Medicine and Health, University of Manchester, Manchester M13 9PL, UK. ${ }^{3}$ School of Clinical Dentistry, University of Sheffield, Claremont Crescent, Sheffield S10 2TA, UK. "King's College London Dental Institute, Bessemer Road, London SE5 9RS, UK.
\end{abstract}

Published online: 01 September 2017

\section{Reference}

1. Development of a core outcome set for orthodontic trials using a mixedmethods approach: protocol for a multicentre study. Trials. 2017;18:366. doi:10.1186/s13063-017-2098-x.

\footnotetext{
*Correspondence: a.tsichlaki@qmul.ac.uk

${ }^{1}$ Department of Orthodontics, Barts and the London School of Medicine and Dentistry, Queen Mary University of London, London E1 1BB, UK
} 